Research Paper

\title{
Proguanil synergistically sensitizes ovarian cancer cells to olaparib by increasing DNA damage and inducing apoptosis
}

\author{
Yan $\mathrm{Wu}^{1}$, Tianyu $\mathrm{Wu}^{1}$, Xin $\mathrm{Hu}^{2}$, Simeng $\mathrm{Xu}^{2}$, Di Xiao ${ }^{2}$, Jingtao $\mathrm{Wu}^{2}$, Xinjian $\mathrm{Yan}^{1}$, Xiaoping $\mathrm{Yang}^{2}{ }^{\circledR}$ and \\ Gaofeng Li1 ${ }^{\circledR}$ \\ 1. Department of Oncology, Zhuzhou Hospital Affiliated to Xiangya School of Medicine, Central South University, Zhuzhou 412000, Hunan, China. \\ 2. Key Laboratory of Study and Discovery of Small Targeted Molecules of Hunan Province, Department of Pharmacy School of Medicine, Hunan Normal \\ University, Changsha 410013, Hunan, China. \\ $\triangle$ Corresponding authors: Gaofeng Li, Zhuzhou Central Hospital, Central South University, Zhuzhou 412000, Hunan, China. E-mail: 2921723380@qq.com; Tel: \\ 01186-139-7532-2472; Xiaoping Yang, Department of Pharmacy, School of Medicine, Hunan Normal University, Changsha 410013, Hunan, China. E-mail: \\ Xiaoping.Yang@hunnu.edu.cn; Tel: 01186 -158-7406-6132. \\ () The author(s). This is an open access article distributed under the terms of the Creative Commons Attribution License (https://creativecommons.org/licenses/by/4.0/). \\ See http://ivyspring.com/terms for full terms and conditions.
}

Received: 2021.09.12; Accepted: 2021.12.16; Published: 2022.01.01

\begin{abstract}
Ovarian cancer is the second leading cause of cancer-related deaths in women, with low five-year survival rates. Therefore, it is essential to seek new treatment options. Olaparib, a PARP inhibitor, has benefited many ovarian cancer patients, but olaparib is much less effective as a single agent in $50 \%$ of patients with high grade severe tumors. Proguanil, which was originally developed as an anti-malarial drug, has gained attention due to its anti-tumor effects. Here, we evaluated the anti-tumor effect of the combination of olaparib and proguanil on ovarian cancer cells, aimed to develop a potential medical option for treating ovarian cancer patients. We examined the effect on proliferation by MTT and colony formation assays, while cell migration was measured by the transwell assay. The effect on apoptosis was measured by flow cytometry and AO/EB staining assays. Western blotting was used to detect protein expression levels in cells treated with olaparib and/or proguanil. In addition, the synergistic effect of these two drugs is calculated by CompuSyn software. The combination of olaparib and proguanil significantly increased growth suppression and apoptosis in ovarian cancer cells, compared to either single agent alone. Furthermore, results showed that the combination of olaparib and proguanil synergistically increased olaparib-induced apoptosis and DNA damage and reduced the efficiency of DNA homologous recombination repair. Our findings indicate that combination of olaparib with proguanil will be a novel potential administration route for treating ovarian cancer patients.
\end{abstract}

Key words: Proguanil; olaparib; ovarian cancer; combination therapy

\section{Introduction}

Ovarian cancer is a gynecological tumor with a high mortality rate, second only to breast cancer in gynecological tumors. Currently, there are nearly 13770 deaths and 21410 new cases of ovarian cancer in the United States every year [1]. The main treatment methods are surgical resection and combination with various chemotherapies, but the recurrence of patients and drug resistance make the treatment prognosis poor. The five-year survival rate of advanced ovarian cancer has not been improved significantly for many years [2]. Therefore, the development of new treatment is very urgent.

Olaparib became the first PARP inhibitor approved by FDA for treating BRCA1/2 mutated ovarian cancer patients who are receiving three or more chemotherapy regimens in 2014. The mechanism in the treatment of ovarian cancer is mainly through inhibiting homologous recombination repair, increasing DNA damage, realizing synthetic lethal and killing tumor cells [3]. Although olaparib has achieved good clinical response, only about 50\% of ovarian cancers are homologous recombination 
(HR) deficient, which makes PARP inhibitors ineffective to considerable amounts of ovarian cancer patients [4]. Developing strategies to extend the use of PARP inhibitor to HR-proficient oncology is a key clinical challenge. One strategy is to develop rational combinations of PARPI with other drugs.

Proguanil, a member of the biguanide family, was originally developed as an anti-malarial drug [5]. Interestingly, we noticed that some studies reported that proguanil may exert anticancer effects by reducing tumor hypoxia, inducing mitochondrial dysfunction and oxidative stress, and causing DNA damage [6]. Moreover, compared with other biguanide drugs, proguanil continued to have the higher inhibitory effect on colon and bladder cancer cell growth [7]. In other hand, the ability of proguanil to sensitize tumor cells to small molecular targeted drugs has not been explored and this area is worthy of deep exploration. Herein, we are trying to investigate the anti-tumor effects of proguanil in combination with olaparib on ovarian cancer cells, which may provide a new option for ovarian cancer treatment.

\section{Materials and Methods}

\section{Reagents}

Olaparib (AZD2281, Ku-0059436) was obtained from Targetmol (Shanghai, China). Proguanil was obtained from Selleck Chemicals (Shanghai, China). Acridine orange/ethidium bromide (AO/EB) staining was obtained from Kehang Biotechnology (Changsha, China), and the FITC Annexin V Apoptosis Detection kit was from BD Pharmingen (NJ, USA). Rabbit anti-human antibodies specific for the following proteins were obtained from Cell Signaling (MA, USA): Phospho-Histone H2A.X (Ser139) and $\beta$-actin. Phospho-ATM (Ser1987) was obtained from Abcam (MA, USA). BRCA1 (sc-6954) was obtained from Santa Cruz Biotechnology (Texas, USA). Bcl-2 polyclonal antibody and Bax polyclonal antibody were obtained from Bioworld Technology (MN, USA) and Biyotime (Shanghai, China) respectively.

\section{Cell culture}

Human ovarian cancer SKOV3 and OVCAR3 cell lines provided by Xiangya Hospital Affiliated to Central South University (Changsha, Hunan, China) were cultured in RPMI-1640 (HyClone) medium supplemented with $10 \%$ fetal bovine serum (Hyclone) and $1 \%$ penicillin-streptomycin (Hyclone) at $37^{\circ} \mathrm{C}$ in a humidified atmosphere with $5 \% \mathrm{CO}_{2}$.

\section{MTT assay}

MTT assay was used to detect the viability of ovarian cancer cells. Cells were added to 96 well plates $\left(6 \times 10^{3}\right.$ cells/well) for $24 \mathrm{~h}$, then olaparib and/or proguanil of different concentrations were treated for $72 \mathrm{~h}$. Then $2 \mathrm{mg} / \mathrm{mL}$ MTT tetrazolium salt (Sigma) (50 $\mu \mathrm{L})$ was added to each well for 5 hours, then $150 \mu \mathrm{L}$ of DMSO (Sigma) was added to each well. The absorbance of each well was measured at $490 \mathrm{~nm}$ using a microplate reader (BioTek, Synergy HTX, Vermont, USA).

\section{Calculation of Combination index ( $\mathrm{Cl})$}

On the basis of the results of the MTT assay, CI values were calculated using CompuSyn software according to the Chou-Talay method [8]. A CI level of $<0.9, \mathrm{CI}=0.9-1.1$ and $\mathrm{CI}>1.1$ indicates synergistic, additive and antagonistic effects of the two drug combinations, respectively [9].

\section{Colony formation assay}

Colony formation was used to monitor the proliferation of ovarian cancer cells. Cells were placed in a 24 -well plate at $5 \times 10^{3} /$ well and then treated with a series of olaparib and/or proguanil doses for $24 \mathrm{~h}$ and collected after 5-7 days. Next, the culture medium is discarded. Cells were carefully washed twice with PBS and fixed with $10 \%$ formalin for $2 \mathrm{~h}$. Add $0.1 \%$ crystal violet solution to the dye and treat for 2 hours. The absorbance at $550 \mathrm{~nm}$ was measured by a microplate reader (BioTek, Synergy HTX, Vermont, USA).

\section{Transwell migration assay}

Transwell assay was employed to evaluate the migration of ovarian cancer cells. $5 \times 10^{4}$ cells were placed in $200 \mu \mathrm{l}$ serum-free medium and added into the upper chamber, and the medium containing $10 \%$ fetal bovine serum was added into the lower chamber. Then appropriate olaparib and/or proguanil were added for $24 \mathrm{~h}$, after which the cells in the upper compartment were removed, the remaining cells were fixed with $10 \%$ formaldehyde for $30 \mathrm{~min}$, stained with $0.1 \%$ crystal for $2 \mathrm{~h}$, and then imaged by microscope (Leica, DFC450C; Wetzlar, Germany). The number of migrated cells in three random fields was counted.

\section{Flow Cytometry Annexin V-FITC-PI Assay}

Apoptotic cell death was assessed by Annexin V-FITC/PI double staining. In brief, ovarian cancer cells were placed in a 6 -well plate at $5 \times 10^{5}$ cells/well and treated with different doses of olaparib and proguanil for $24 \mathrm{~h}$. Cells were collected and stained with Annexin V-FITC and PI (5 $\mu \mathrm{l}$ each) in a $300 \mu \mathrm{L}$ volume and incubated in darkness for $20 \mathrm{~min}$. The apoptotic cells were then evaluated by FACS Calibur flow cytometer (Becton, Dickinson and Company, New Jersey, USA). 


\section{Microscopy Imaging and Acridine Orange- Ethidium Bromide Staining}

Acridine Orange-Ethidium Bromide Staining was used to observe the cell apoptosis from the changes of cell morphology. Cells were added to 6 well plates $\left(5 \times 10^{5}\right.$ cells/well) for $24 \mathrm{~h}$, then olaparib and/or proguanil of different concentrations were added for $24 \mathrm{~h}$. After that, cells were collected and cleaned with PBS once, and stained with AO/EB working solution for 5-15 min. The apoptotic cells were then observed and photographed via microscope (Leica, DFC450C; Wetzlar, Germany).

\section{Western Blotting}

Whole cell lysates from each sample were loaded onto a $10 \%$ polyacrylamide gel for electrophoresis and transferred to a PVDF membrane. After the primary antibody was incubated overnight at $4{ }^{\circ} \mathrm{C}$, it was washed with PBS/0.1\% Tween-20, and then incubated at room temperature with the secondary antibody for 1 hour. The protein was visualized using Clarity Western ECL Substrate (Bio-Rad, California, USA) and imprinted immediately on the Chemidoc system (Tanon 4600, Shanghai, China). Protein expression was quantified using Image J.

\section{Statistical analyses}

Data are expressed as \pm standard deviation. Two independent groups were compared using twotailed t-tests and two-way ANOVAs. All statistical analyses were performed within $95 \%$ confidence intervals and the corresponding $P$-values were reported $\left({ }^{*} \mathrm{P}<0.05,{ }^{* *} \mathrm{P}<0.01,{ }^{* * *} \mathrm{P}<0.001\right)$. Statistical analysis was performed using GraphPad Prism 6 and SPSS 25.0.

\section{Results}

\section{Cytotoxicity of the combination of olaparib and proguanil on ovarian cancer cells}

In this study, we evaluated the antitumor effect of the combination of olaparib and proguanil on human ovarian cancer cell lines, including OVCAR-3 and SKOV-3. Cells were treated with a serial concentration of olaparib, proguanil or both for 72 hours, and the survival rate of each treatment group was detected using MTT assay. We found different drug sensitivities in these two types of cells, which provided a reference for the selection of concentrations in our subsequent series of experiments. Dose-dependent inhibition of proliferation of both cell types by both drugs after 72 hours was witnessed, with estimated $\mathrm{IC}_{50}$ values of $21.7 \mathrm{umol} / \mathrm{L}$ (OVCAR-3) and $80.9 \mathrm{umol} / \mathrm{L}$ (SKOV-3) for olaparib, and $22.4 \mathrm{umol} / \mathrm{L}$ (OVCAR-3) and 45.7
umol/L (SKOV-3) for proguanil. Based on this finding, different drug concentrations were used for the two cell lines in subsequent experiments. The combination of the two drugs had an enhanced cell growth inhibitory effect, compared to each drug alone (Fig. 1A). The combination index of the two drugs was calculated by using CompuSyn software to determine whether the two drugs have synergistic effects. The level of CI for OVCAR-3 ranged from 0.726 to 0.991 and for SKOV-3 varied from 0.433 to 0.8 , shown in Fig. 1B. Most $\mathrm{CI}$ values for OVCAR-3 and all CI values for SKOV-3 were less than 0.9, demonstrating a synergistic effect of the two drugs. A small minority of CI values for OVCAR-3 fell within 0.9 and 1, which represented a simple additive effect.

Next, we assessed whether the combination of olaparib and proguanil induced long-term synergistic effects in anti-tumor activity by colony formation. This assay showed that the combination of olaparib and proguanil was effective in inhibiting ovarian cancer cell colony formation (Fig. 1C-D). Taken together, the combination of olaparib and proguanil showed more potent antitumor effects against two human ovarian cancer cell lines.

\section{Olaparib and proguanil inhibit the migration of ovarian cancer cells}

Then, we used the transwell assay to assess the ability of olaparib and proguanil on inhibiting the migration of OVCAR-3 cells and SKOV-3, and the results showed that the combination of these two drugs was able to inhibit this migratory activity, much stronger than either drug alone (Fig. 2A-B).

\section{The combination of olaparib and proguanil results in enhanced apoptosis in ovarian cancer cells}

To further evaluate the synergistic antitumor effects of the two drugs, we also monitored the apoptosis of OVCAR-3 cells and SKOV-3 cells after 24 hours drug treatment. Flow cytometry was used to detect cell apoptosis with Annexin V-FITC/PI double staining solution and the results showed that the combination of the two drugs enhanced cell apoptosis in ovarian cancer cells, compared monotherapy (Fig. 2C-D).

To further confirm the effect of the combination of the two drugs on apoptosis, we used the AO/EB staining assay to detect this effect. Unlike flow cytometry, where the principle of detection is to measure the apoptosis-related alterations of cell membrane, AO/EB double staining is to detect the apoptosis-related difference of nucleic acids including the binding of single DNA strand, double DNA strand and RNA. OVCAR-3 cells and SKOV-3 cells 
were stained with $\mathrm{AO} / \mathrm{EB}$ and typical pictures were shown (Fig. 3A-B). In the drug combination group,

A

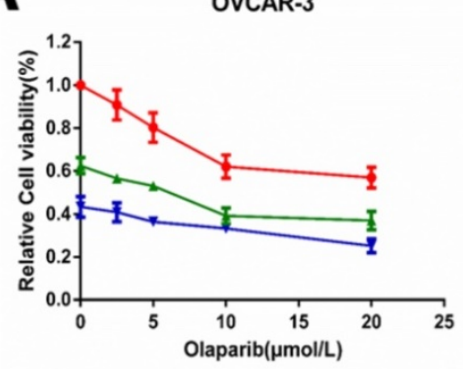

SKOV-3

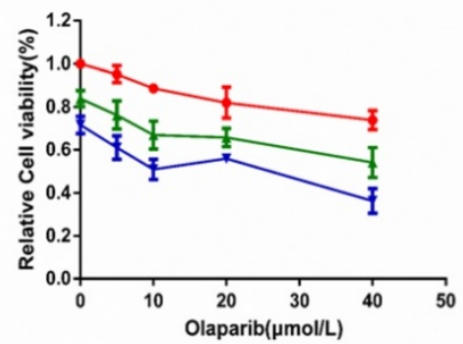

C

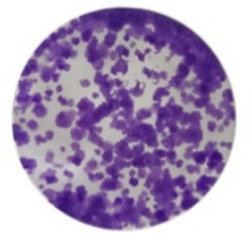

- Proguanil(Oumol/L)

$\star$ Proguanil(10umol/L)

* Proguanil(20umol/L)

$\rightarrow$ Proguanil(Oumol/L)

‡ Proguanil(10umol/L)

- Proguanil(20umol/L) more apoptotic and necrotic cells could be observed than in the single drug-treated.

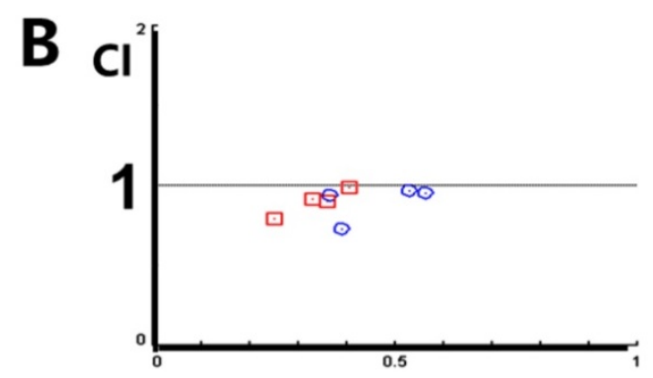

Fa

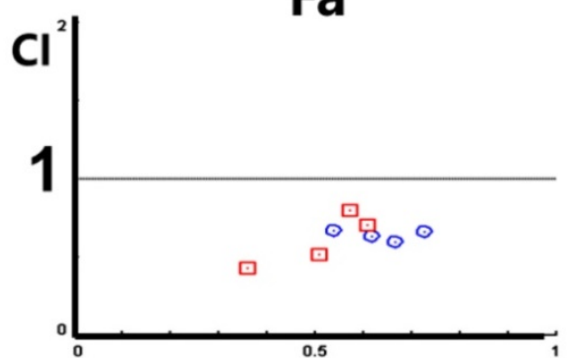

$\begin{array}{ll}\circ & 0+p 10 \\ 0 & 0+p 20\end{array}$
OVCAR-3

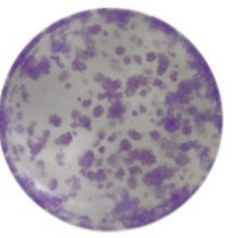

$+$

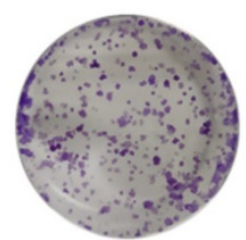

Fa

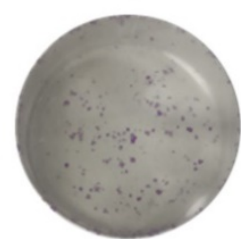

$+$

$+$

\section{SKOV-3}
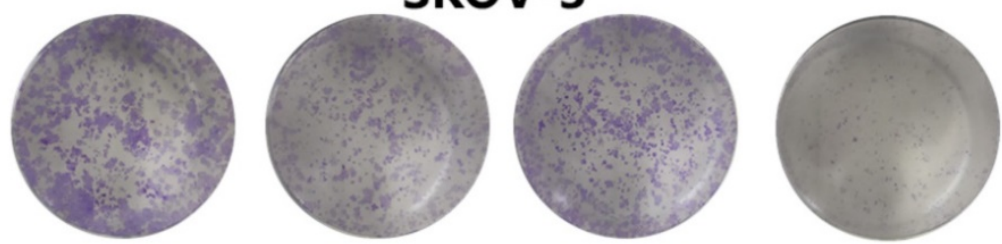

\section{Ola(20umol/L)}

Pro(10umol/L)

D
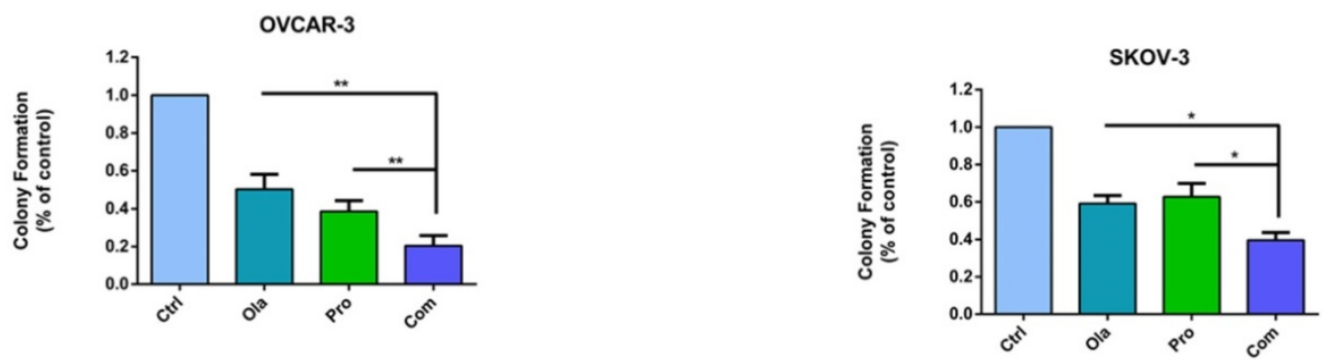

Figure 1. Effects of olaparib combined with proguanil on proliferation and colony-forming activity of OVCAR-3 and SKOV-3 cells. (A) Olaparib and proguanil synergistically inhibited cell proliferation of OVCAR-3 and SKOV-3 cells, which was measured at $72 \mathrm{~h}$ post-treatment with olaparib and/or proguanil. (B) Synergy between two 
drugs is evaluated by the combination index $(\mathrm{Cl})$, with $\mathrm{Cl}=0.9-1.1, \mathrm{Cl}>1.1$ and $\mathrm{Cl}<0.9$ indicating additive, antagonistic and synergistic effects respectively. In the majority of cases, $\mathrm{Cl}$ values were below 0.9 , indicating a synergistic effect of two drugs. (C) Evaluation of the ability of olaparib and proguanil to suppress colony formation. Cells were incubated with olaparib and/or proguanil for 5-7 days and then photographed. (D) The colony-formation ability of each group was shown in bar chart and described as mean \pm s.d of three independent experiments. (*P $<0.05, * * \mathrm{P}<0.01, \mathrm{n}=3$ )

\section{A}

\section{OVCAR-3}

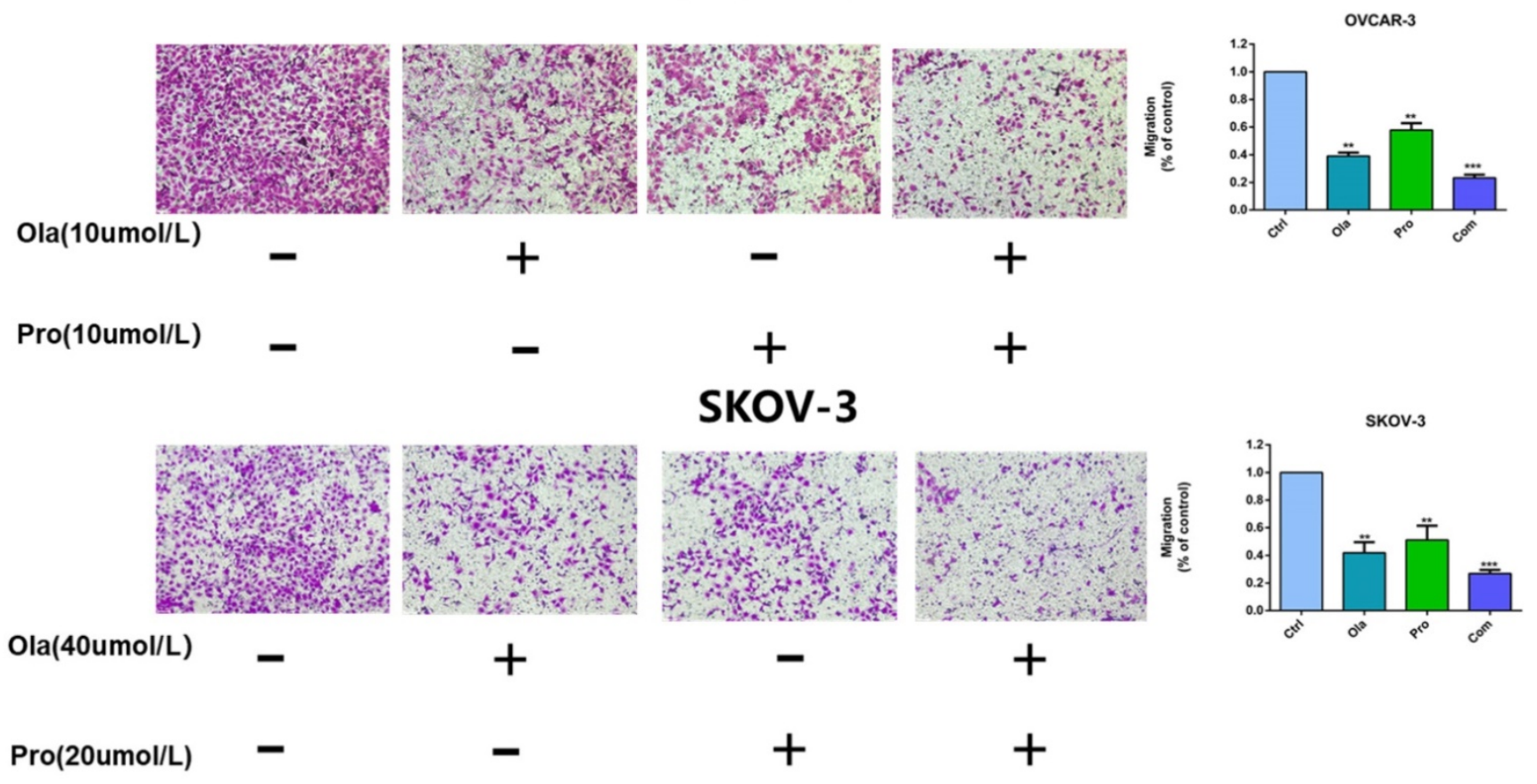

C

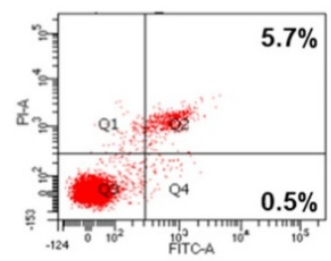

Ctrl

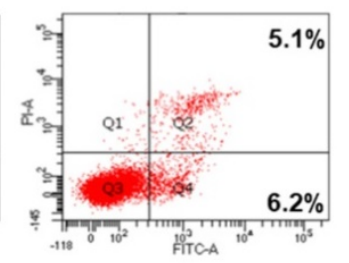

Olaparib(10umol/L)

\section{OVCAR-3}

\section{SKOV-3}
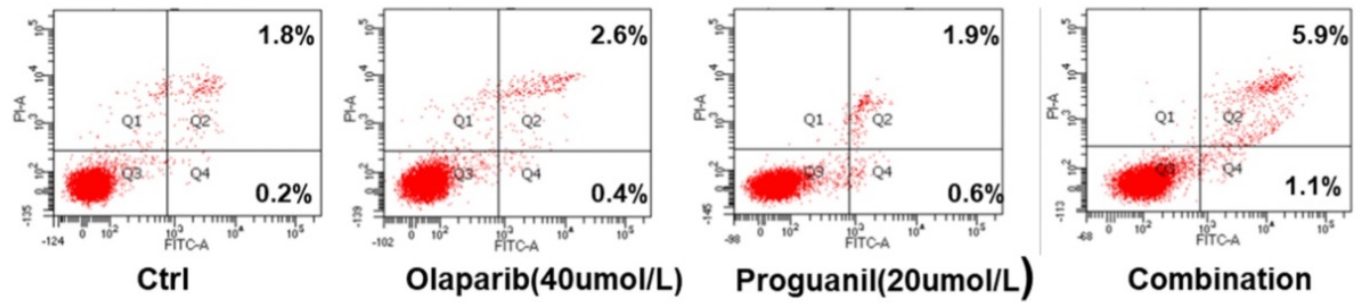

D
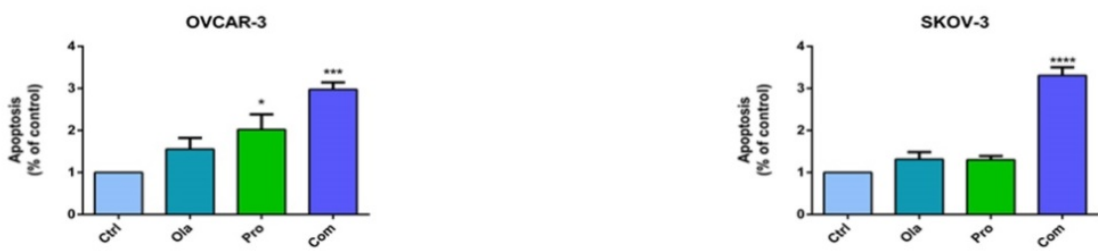

Figure 2. Effects of olaparib combined with proguanil on migration and apoptosis of OVCAR-3 and SKOV-3 cells. (A) Combination of olaparib and proguanil treatment suppressed the migratory activity of OVCAR-3 and SKOV-3 cells in $\mathrm{n}$ a transwell assay. (B) Quantification results were presented. (C) Representative flow cytometry plots corresponding to cells stained with propidium iodide ( $y$-axis) and Annexin V-FITC ( $x$-axis). (D) Quantification results were presented. ( $* \mathrm{P}<0.05$, $* * \mathrm{P}<0.01, * * * \mathrm{P}<0.001$, $\mathrm{n}=3)$. 
A

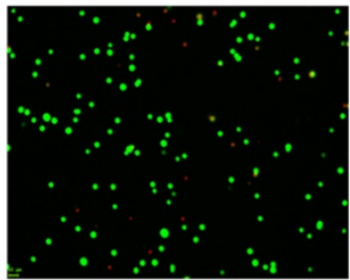

Ctrl

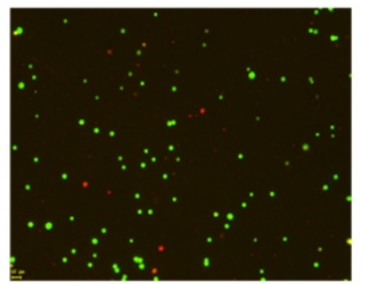

Olaparib(10umol/L)
Proguanil(10umol/L)

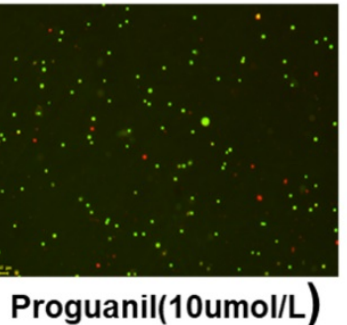

SKOV-3

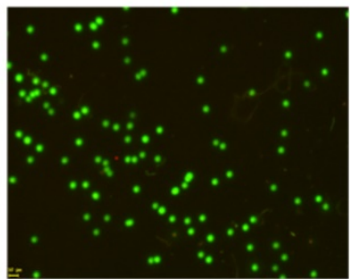

Ctrl

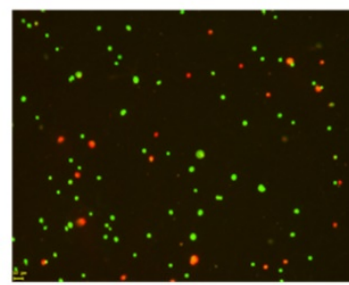

Olaparib(40umol/L)

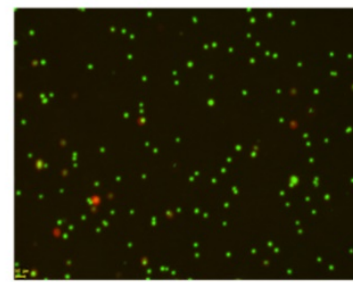

Proguanil(20umol/L)

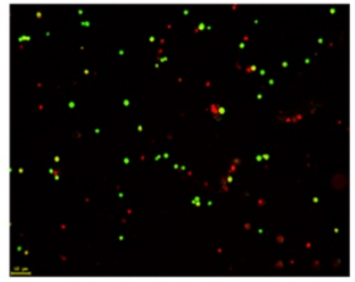

Combination

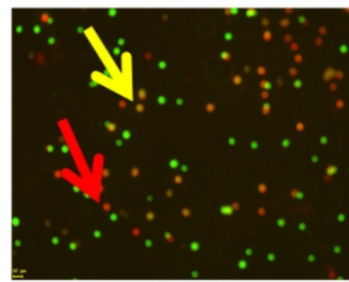

Combination
B

C

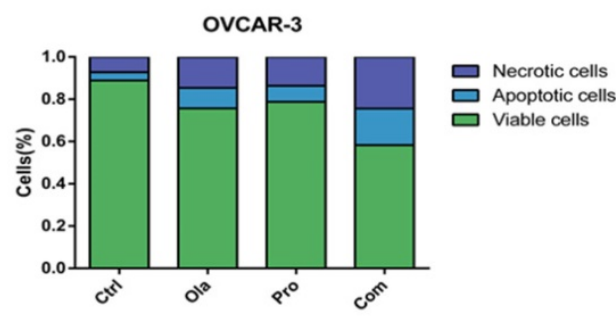

OVCAR-3

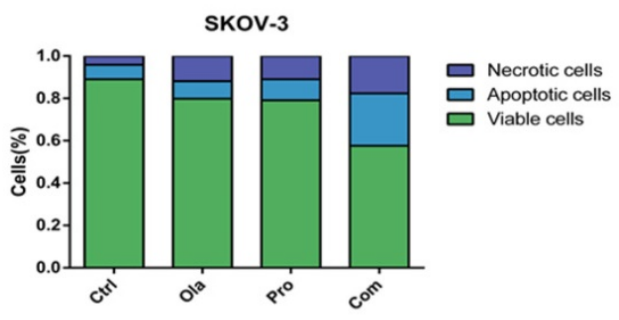

\section{SKOV-3}

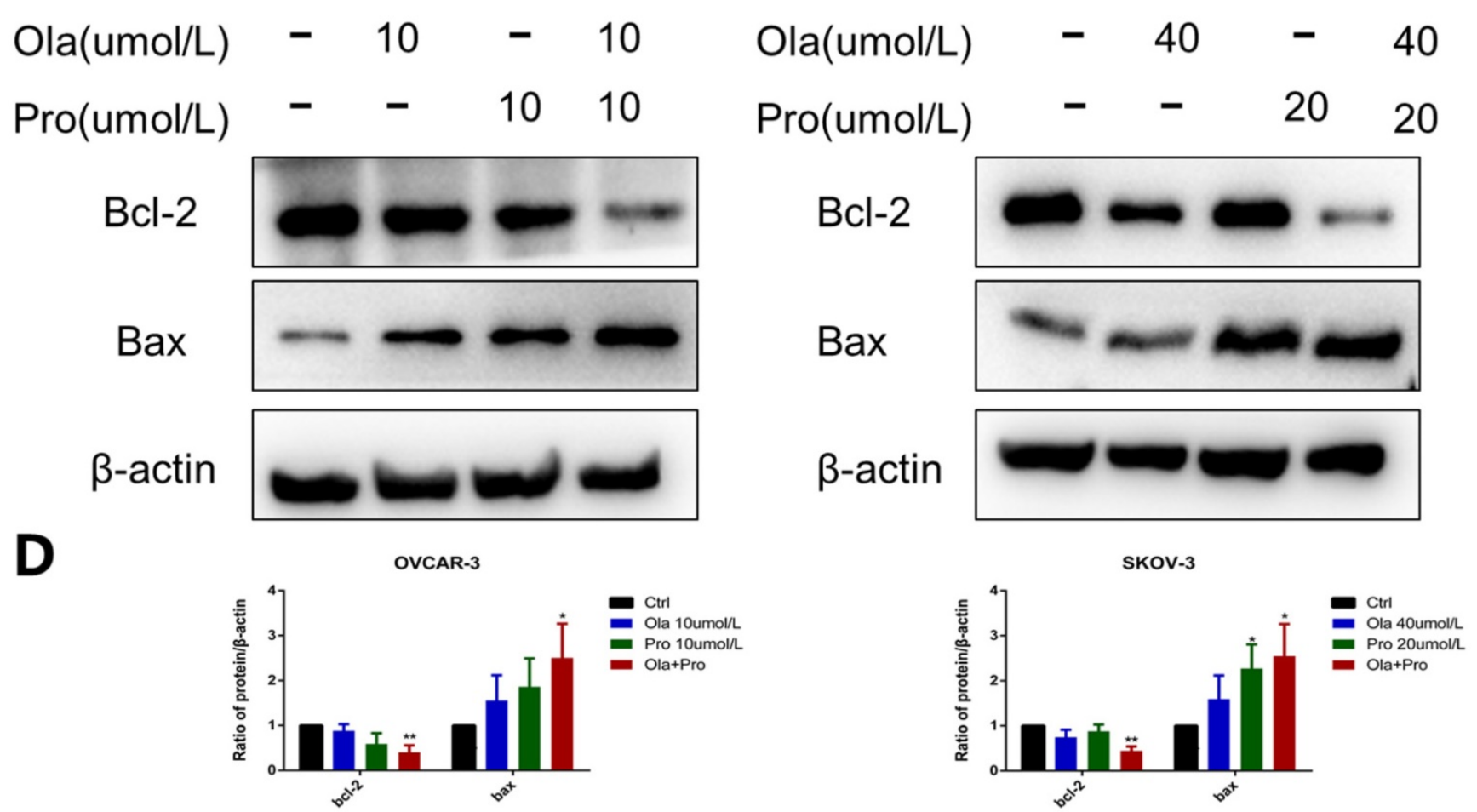

Figure 3. Olaparib and proguanil synergistically enhance apoptosis of OVCAR-3 and SKOV-3 cells. (A) AO/EB staining assay in ovarain cells treated with olaparib and/or proguanil was used to examine cell death. The yellow arrows indicate apoptotic cells, which are yellow or orange. And red arrows indicate necrotic cells, which are red. (B) Quantification results were presented. (C) The effects of combination treatment of olaparib and proguanil on the protein expression of Bcl-2 and Bax were analyzed by western blotting. (D) Relative levels of various proteins. ( $* P<0.05, * * P<0.01$, *** $P<0.001, n=3$.) 
The Bcl-2 family has an essential role in monitoring the mitochondrial apoptosis pathway [10]. Within Bcl-2 family, two most prominent molecules are Bcl-2 and Bax. Bcl-2 is a known anti-apoptotic protein, while Bax is assumed to be a pro-apoptotic protein. It is well known that the intracellular Bcl-2/Bax ratio is a critical factor in determining the susceptibility to apoptosis [11]. Our results show that the decrease of Bcl-2 expression and the increase of Bax expression were significantly detected in ovarian cancer cells treated with the combination of olaparib and proguanil (Fig. 3C-D). The decreasing bcl-2/bax ratio represents an increase in susceptibility to apoptosis, as shown in Figure S1, which was consistent with the results stated above. These results suggested that proguanil enhances olaparib-induced apoptosis associated with mitochondria. This observation is consistent with literature which reported that proguanil enhanced the action of atovaquone, causing mitochondrial membrane potential disintegration and leading to mitochondrial dysfunction [12].

\section{The combination of olaparib and proguanil induces increased DNA damage by reducing homologous recombination repair in ovarian cancer cells}

It is well documented that p-ATM and $\gamma \mathrm{H} 2 \mathrm{AX}$ are important molecular markers of DNA damage [13, 14], while BRCA1 is an essential protein to support DNA homologous recombination repair [15]. To further explore the mechanisms underlying, we used western blotting to examine p-ATM, $\gamma \mathrm{H} 2 \mathrm{AX}$, and BRCA1 protein levels in cells after these different treatments. Consistent with previous observations [16], olaparib alone increased the expression level of p-ATM and $\gamma \mathrm{H} 2 \mathrm{AX}$, and decreased the expression level of BRCA1 (Fig. 4A-B). In contrast, compared with olaparib alone, the effect of proguanil on DNA damage and inhibition of homologous recombination repair was not very strong yet (Fig. 4C-D). Proguanil merely slightly increased the expression levels of p-ATM and $\mathrm{YH} 2 \mathrm{AX}$, and modestly decreased the expression level of BRCA1. However, when two drugs were combined, these effects were much stronger than that of either single drug treatment alone. There was a more remarked increase in p-ATM and $\gamma \mathrm{H} 2 \mathrm{AX}$ in the combination group, and a more profound decrease in BRCA1 (Fig. 4E-F). Together, these results demonstrated that proguanil enhanced olaparib-induced DNA damage-related events, and the decrease in BRCA1 expression may reduce the efficiency of DNA homologous recombination repair.

\section{Discussion}

In this study, we demonstrate for the first time that proguanil can sensitize ovarian cancer cells to the small molecular-targeted drug olaparib. Our results suggest that proguanil and olaparib can exert a notable synergistic antitumor effect on ovarian cancer cells. The mechanisms underlying may be related to the increase of olaparib-induced apoptosis and DNA damage, and reduce of the efficiency of DNA HR repair.

Ovarian cancer has a long history of being a highly lethal tumor of the female reproductive system [2]. Olaparib, the first-in-class PARP inhibitor for the treatment of ovarian cancer patients, has benefited many ovarian patients [3]. One clinical study showed that in patients with platinum-resistant BRCA1/2 mutation ovarian cancer, olaparib as maintenance monotherapy showed a progression-free survival (PFS) of 11.2 months compared to 4.3 months with placebo, a dramatic improvement for treating ovarian cancer patients [17]. However, this benefit is very limited clinically due to limitations in the scope of targeted patients, high recurrence rate and drug resistance [18-21]. Therefore, it is an urgent need to pursue new treatment options.

Proguanil, originally developed as an antimalarial drug [5], has been documented with good anti-proliferative effects [7]. Compared to metformin, another biguanide compound, the effective antitumor concentration of proguanil is considerably low [7], and it has no side effect of lactic acidosis which appears on other biguanide drugs [22]. In this study, we for the first time explored the combinational effect of proguanil with olaparib on ovarian cancer. DNA is constantly damaged by environmental attacks and activities of endogenous metabolism [23]. DNA damage response is composed of five major complementary pathways that enhance genome integrity and stability [24]. HR repair is the main pathway for repairing double-strand breaks [25], and BRCA1 and 2 are important proteins involved in this pathway, of which mutations in these genes predispose to occurrence of tumors including ovarian cancer [26]. It has been widely recognized that p-ATM and $\gamma \mathrm{H} 2 \mathrm{AX}$ are important molecular markers of DNA damage [27]. In the current study, we mainly measured the changes of the expressions of three proteins: $\mathrm{p}-\mathrm{ATM}, \gamma \mathrm{H} 2 \mathrm{AX}$, and BRCA1. We found that the combination of two drugs increased the expression of $\mathrm{p}-\mathrm{ATM}, \mathrm{\gamma H} 2 \mathrm{AX}$, and decreased the expression of BRCA1. Thus, it is clear that the combination of two drugs increased DNA damage with decrease of the efficiency of HR repair. 


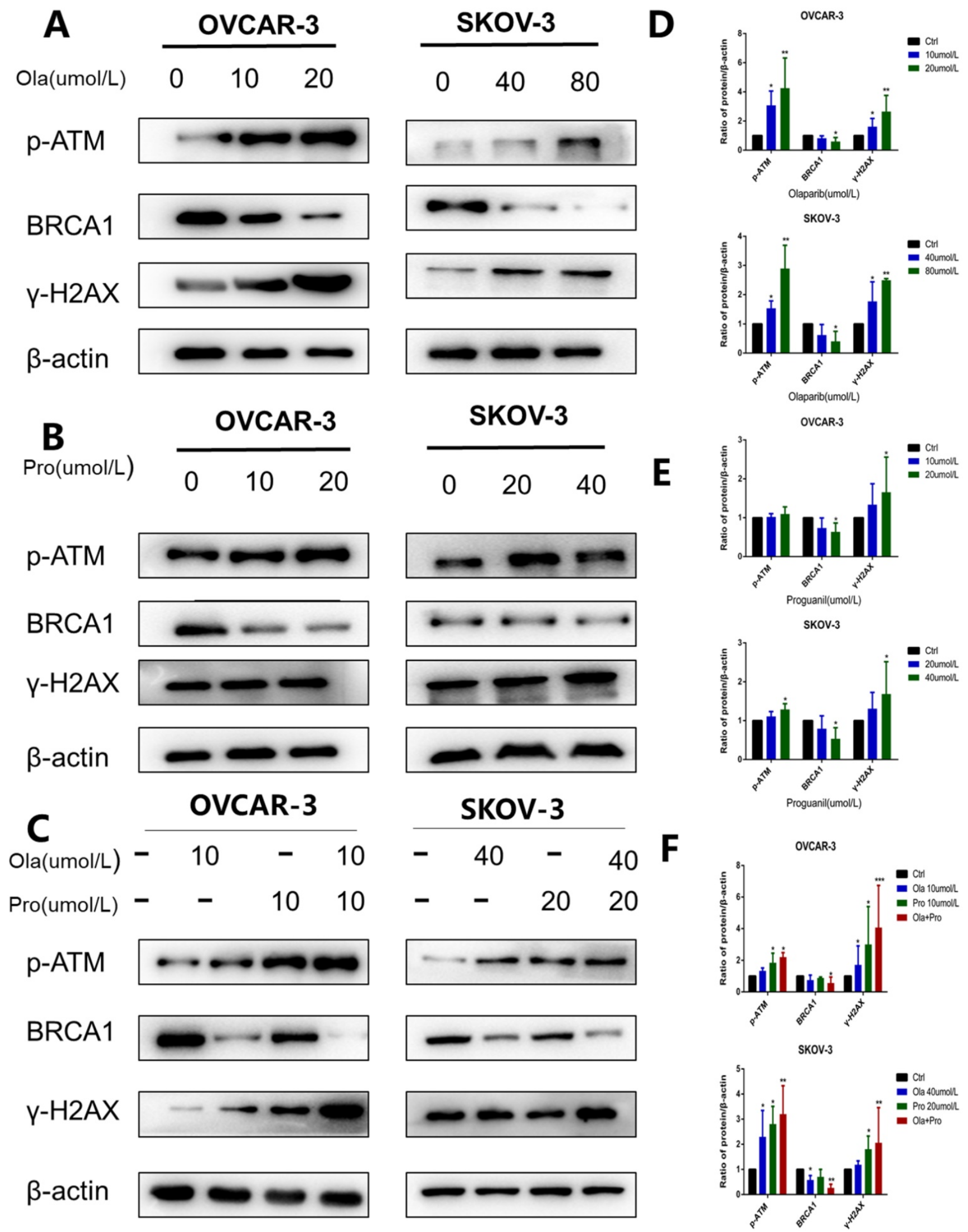

Figure 4. Effects of olaparib and proguanil alone or in combination on homologous recombination repair signaling. Western blotting was used to examine the levels of key proteins associated with the HRR pathway after treatment with olaparib and/or proguanil in OVCAR-3 and SKOV-3 cells. (A-C)The protein expression of p-ATM, $\mathrm{Y}-\mathrm{H} 2 \mathrm{AX}, \mathrm{BRCA} 1$ and $\beta$-actin were measured, using $\beta$-actin as a loading control. ( $\mathrm{D}-\mathrm{F})$ Relative levels of various proteins. (*P $<0.05, * * \mathrm{P}<0.01, * * * \mathrm{P}<0.001, \mathrm{n}=3$.) 
Our study highlights a potentially viable approach for the treatment of ovarian cancer. Wilson et al. suggested that the sensitivity of olaparib can be enhanced by reducing the efficiency of HR repair, therefore targeting this pathway may be a viable antitumor strategy [9]. In the present study, our results suggest that proguanil enhances the ability of olaparib to inhibit the proliferation, migration and survival of ovarian cancer cells. These two drugs may exhibit synergistic anticancer activity by mainly decreasing the efficiency of HR repair and increasing olaparib-induced DNA damage and apoptosis. Therefore, the synergistic antitumor effect of olaparib and proguanil promotes a potentially safe and highly effective treatment option for ovarian cancer by combination of these two drugs.

\section{Abbreviations}

MTT, 3-(4,5-dimethylthiazol) 2: 5-diphenyltetrazolium; AO/EB: Acridine Orange-Ethidium Bromide; HR: homologous recombination; DMSO: dimethyl sulfoxide; PBS: fetal bovine serum; $\gamma \mathrm{H} 2 \mathrm{AX}$ : phospho-Histone H2AX; p-ATM: phospho-ATM; PFS: progression-free survival.

\section{Supplementary Material}

Supplementary figure.

https://www.medsci.org/v19p0233s1.pdf

\section{Acknowledgements}

This work was supported by the National Natural Science Foundation of China (No. 81874212); Huxiang High-Level Talent Innovation Team (2018RS3072); Opening Fund for Key Laboratory of Molecular Pharmacology and Drug Evaluation (Yantai University), Ministry of Education (P201905); Scientific and Technological Projects for Collaborative Prevention and Control of Birth Defect in Hunan Province (2019SK1012) and Key Grant of Research and Development in Hunan Province (2020DK2002).

\section{Author Contributions}

All authors contributed to the design, drafting, and substantive revision of the manuscript.

\section{Competing Interests}

The authors have declared that no competing interest exists.

\section{References}

1. Siegel RL, Miller KD, Fuchs HE et al. Cancer Statistics, 2021. CA Cancer J Clin 2021; 71 (1): 7-33.

2. Reid BM, Permuth JB, Sellers TA. Epidemiology of ovarian cancer: a review. Cancer Biol Med 2017; 14 (1): 9-32.

3. Goulooze SC, Cohen AF, Rissmann R. Olaparib. Br J Clin Pharmacol 2016; 81 (1): $171-173$
4. Konstantinopoulos PA, Ceccaldi R, Shapiro GI et al. Homologous Recombination Deficiency: Exploiting the Fundamental Vulnerability of Ovarian Cancer. Cancer Discov 2015; 5 (11): 1137-1154.

5. Blanshard A, Hine P. Atovaquone-proguanil for treating uncomplicated Plasmodium falciparum malaria. Cochrane Database Syst Rev 2021; 1: CD004529.

6. Mudassar F, Shen H, O'Neill G et al. Targeting tumor hypoxia and mitochondrial metabolism with anti-parasitic drugs to improve radiation response in high-grade gliomas. J Exp Clin Cancer Res 2020; 39 (1): 208.

7. Lea MA, Kim H, des BC. Effects of Biguanides on Growth and Glycolysis of Bladder and Colon Cancer Cells. Anticancer Res 2018; 38 (9): 5003-5011.

8. Chou TC. Drug combination studies and their synergy quantification using the Chou-Talalay method. Cancer Res 2010; 70 (2): 440-446.

9. Wilson AJ, Stubbs M, Liu P et al. The BET inhibitor INCB054329 reduces homologous recombination efficiency and augments PARP inhibitor activity in ovarian cancer. Gynecol Oncol 2018; 149 (3): 575-584.

10. Ashkenazi A, Fairbrother WJ, Leverson JD et al. From basic apoptosis discoveries to advanced selective BCL-2 family inhibitors. Nat Rev Drug Discov 2017; 16 (4): 273-284.

11. Pena-Blanco A, Garcia-Saez AJ. Bax, Bak and beyond - mitochondrial performance in apoptosis. FEBS J 2018; 285 (3): 416-431.

12. Painter HJ, Morrisey JM, Mather MW et al. Specific role of mitochondrial electron transport in blood-stage Plasmodium falciparum. Nature 2007; 446 (7131): 88-91.

13. Jin MH, Oh DY. ATM in DNA repair in cancer. Pharmacol Ther 2019; 203: 107391.

14. Georgoulis A, Vorgias CE, Chrousos GP et al. Genome Instability and gammaH2AX. Int J Mol Sci 2017; 18 (9).

15. Wagener-Ryczek S, Merkelbach-Bruse S, Siemanowski J. Biomarkers for Homologous Recombination Deficiency in Cancer. J Pers Med 2021; 11 (7).

16. Mani C, Jonnalagadda S, Lingareddy J et al. Prexasertib treatment induces homologous recombination deficiency and synergizes with olaparib in triple-negative breast cancer cells. Breast Cancer Res 2019; 21 (1): 104.

17. Ledermann JA, Pujade-Lauraine E. Olaparib as maintenance treatment for patients with platinum-sensitive relapsed ovarian cancer. Ther Adv Med Oncol 2019; 11: 1758835919849753.

18. Brown JS, Kaye SB, Yap TA. PARP inhibitors: the race is on. Br J Cancer 2016; 114 (7): 713-715.

19. Devouassoux-Shisheboran M, Genestie C. Pathobiology of ovarian carcinomas. Chin J Cancer 2015; 34 (1): 50-55.

20. Ventriglia J, Paciolla I, Pisano C et al. Immunotherapy in ovarian, endometrial and cervical cancer: State of the art and future perspectives. Cancer Treat Rev 2017; 59: 109-116.

21. Scott CL, Swisher EM, Kaufmann SH. Poly (ADP-ribose) polymerase inhibitors: recent advances and future development. J Clin Oncol 2015; 33 (12): 1397-1406.

22. Bridges HR, Jones AJ, Pollak MN et al. Effects of metformin and other biguanides on oxidative phosphorylation in mitochondria. Biochem J 2014; 462 (3): 475-487.

23. Roos WP, Thomas AD, Kaina B. DNA damage and the balance between survival and death in cancer biology. Nat Rev Cancer 2016; 16 (1): 20-33.

24. Pearl LH, Schierz AC, Ward SE et al. Therapeutic opportunities within the DNA damage response. Nat Rev Cancer 2015; 15 (3): 166-180.

25. Helleday T, Petermann E, Lundin $C$ et al. DNA repair pathways as targets for cancer therapy. Nat Rev Cancer 2008; 8 (3): 193-204.

26. Farmer H, McCabe N, Lord CJ et al. Targeting the DNA repair defect in BRCA mutant cells as a therapeutic strategy. Nature 2005; 434 (7035): 917-921.

27. Rothkamm K, Barnard S, Moquet J et al. DNA damage foci: Meaning and significance. Environ Mol Mutagen 2015; 56 (6): 491-504. 